\title{
The serotonin reuptake transporter modulates mitochondrial copy number and mitochondrial respiratory complex gene expression in the frontal cortex and cerebellum in a sexually dimorphic manner
}

\author{
Bryony N. Thorne $^{1}$ | Bart A. Ellenbroek ${ }^{2}$ | Darren J. Day ${ }^{1}$
}

${ }^{1}$ School of Biological Sciences, Victoria University of Wellington, Wellington, New Zealand

${ }^{2}$ School of Psychology, Victoria University of Wellington Faculty of Science,

Wellington, New Zealand

\section{Correspondence}

Darren J. Day, School of Biological Sciences, Victoria University of Wellington, Kelburn Parade, Wellington, 6012, New Zealand.

Email: Darren.day@vuw.ac.nz

Funding information

Neurological Foundation of New Zealand; Wellington Medical Research Foundation

\begin{abstract}
Neuropsychiatric and neurodevelopmental disorders such as major depressive disor$\operatorname{der}(\mathrm{MDD})$ and autism spectrum disorder (ASD) are complex conditions attributed to both genetic and environmental factors. There is a growing body of evidence showing that serotonergic signaling and mitochondrial dysfunction contribute to the pathophysiology of these disorders and are linked as signaling through specific serotonin $(5-\mathrm{HT})$ receptors drives mitochondrial biogenesis. The serotonin transporter (SERT) is important in these disorders as it regulates synaptic serotonin and therapeutically is the target of selective serotonin reuptake inhibitors which are a major class of anti-depressant drug. Human allelic variants of the serotonin transporter-linked polymorphic region (5-HTTLPR) such as the $\mathrm{S} / \mathrm{S}$ variant, are associated with reduced SERT expression and increased susceptibility for developing neuropsychiatric disorders. Using a rat model that is haploinsufficient for SERT and displays reduced SERT expression similar to the human S/S variant, we demonstrate that reduced SERT expression modulates mitochondrial copy number and expression of respiratory chain electron transfer components in the brain. In the frontal cortex, genotype-related trends were opposing for males and females, such that reduced SERT expression led to increased expression of the Complex I subunit mt-Nd1 in males but reduced expression in females. Our findings suggest that SERT expression and serotonergic signaling have a role in regulating mitochondrial biogenesis and adenosine triphosphate (ATP) production in the brain. We speculate that the sexual dimorphism in mitochondrial abundance and gene expression contributes to the sex bias found in the incidence of neuropsychiatric disorders such as MDD and ASD.
\end{abstract}

KEYWORDS

mitochondrial biogenesis, neuropsychiatric disorders, serotonin, SERT

\section{1 | INTRODUCTION}

Neuropsychiatric and neurodevelopmental disorders such as major depressive disorder (MDD), autism spectrum disorder (ASD), and schizophrenia show altered neuronal and behavioral plasticity that can be attributed to both genetic and environmental factors. Among the range of molecular processes associated with these disorders, serotonergic signaling and mitochondrial dysfunction are 
strongly implicated (Ferrari \& Villa, 2017; Hollis et al., 2017; Muller et al., 2016). Serotonin (5-hydroxytryptamine (5-HT)) has a longstanding association with neuropsychiatric disorders as it functions in the central nervous system both as a neurotrophin during development and a neurotransmitter with an important role in mood regulation in the adult (Yohn et al., 2017). The serotonin transporter (SERT) regulates synaptic levels of $5-\mathrm{HT}$ by facilitating reuptake of serotonin into the presynaptic terminal, and is an important pharmacological target for the treatment of mood disorders, with inhibition by selective serotonin reuptake inhibitors (SSRIs) serving as a first-line treatment for many disorders (Levinstein \& Samuels, 2014). Genetic variants of SERT have been highlighted as risk factors for neuropsychiatric disorders and while the specificities of these genetic associations are controversial, altered SERT expression and serotonergic signaling remains strongly associated with neuropsychiatric and neurodevelopmental disorders (Border et al., 2019; Brown \& Harris, 2008).

Mitochondria have a critical cellular role in energy transduction, with the brain having an extremely high demand for energy making it particularly sensitive to impaired mitochondrial function. Mitochondrial dysfunction is associated with the pathophysiology of many neuropsychiatric disorders and there is a high incidence of psychiatric symptoms preceding the diagnosis of mitochondrial disease (Allen et al., 2018; Fattal et al., 2006). Impaired mitochondrial function has been shown in both ASD and MDD, which is characterized by reduced glucose metabolism as well as impaired electron transport and adenosine triphosphate (ATP) production in the brain (Klinedinst \& Regenold, 2015; Siddiqui et al., 2016; Streck et al., 2014; Videbech, 2000). The maintenance of synaptic connections and neurotransmission is an energy intensive process in which mitochondrial activity and trafficking are important (Bansal \& Kuhad, 2016; Rossi \& Pekkurnaz, 2019). A reduction in synaptic plasticity and increased neuronal cell death has been implicated in the pathophysiology of MDD (Kraus et al., 2017) and is associated with reduced hippocampal volume (Campbell et al., 2004; MacQueen et al., 2003).

Growing evidence suggests that serotonergic signaling and mitochondrial dysfunction may be linked in the pathophysiology of neuropsychiatric disorders. Mitochondrial biogenesis is regulated by signaling through specific 5-HT receptors (Chaji et al., 2021; Gibbs et al., 2018; Gressier et al., 2016; Lesch et al., 1996; Simmons et al., 2019) with signaling through the $5-\mathrm{HT}_{2 \mathrm{~A}}$ receptor leading to increased expression of the transcriptional coactivator peroxisome proliferator-activated receptor gamma cofactor 1-alpha (PGC-1 $\alpha$ ), in cultured cortical neurons, resulting in increased oxygen consumption, ATP production, mtDNA mass, and antioxidant capacity (Fanibunda et al., 2019). 5 $\mathrm{HT}_{1 \mathrm{~F}}$ receptor agonists have similarly been shown to induce mitochondrial biogenesis in rodent studies of kidney injury, spinal cord injury, and Parkinson's disease (Fanibunda et al., 2019; Gibbs et al., 2018; Scholpa et al., 2018; Simmons et al., 2019). Additionally, stimulation of the $5-\mathrm{HT}_{7}$ receptor has been shown to rescue impaired ATP production in the brains of a rat model of Rett syndrome, further supporting a potential role of serotonergic signaling in the regulation of mitochondrial bioenergetics (Valenti et al., 2017).

The 5-HTTLPR S-allele (S/S genotype) results in around a 50\% reduction in SERT expression and an increased likelihood of developing depressive and anxiety disorders, particularly in women (Gressier et al., 2016; Lesch et al., 1996). The SERT knockout rat has been extensively characterized (Chaji et al., 2021; Homberg et al., 2008; Homberg, Olivier, et al., 2007; Olivier et al., 2008; Smits et al., 2006) and is a valuable model for studying serotonergic signaling, with the heterozygous animals being proposed as a good model of the low expressing human S/S variant (Homberg, Olivier, et al., 2007; Olivier et al., 2008). Given the importance of serotonergic signaling in mitochondrial biogenesis and neuropsychiatric disorders, we sought to determine whether there were differences in mitochondrial abundance and mRNA expression of the mitochondrial respiratory complex subunits in the frontal cortex (FC) and cerebellum (Cb) of SERT knockout wild-type (WT) and heterozygous (HET) rats.

\section{2 | RESULTS}

We first sought to validate the rat SERT heterozygotes as being a suitable model for the human low expressing S/S variant. High resolution melt analysis of DNA and cDNA prepared from the dorsal raphe nuclei from WT, HET, and HOM rats confirms that the HET animals contain approximately $70 \%$ less SERT transcript than WT animals, and the HOM animals contain minimal transcript (Figure S1).

To determine whether the differences in SERT expression resulted in changes in expression of the mitochondrial encoded respiratory complex genes and the nuclear encoded subunits for male and female animals, a conventional quantitative reverse-transcription PCR (qRT-PCR) approach using reference housekeeping genes was undertaken. qRT-PCR is a routinely used technique for analysis of gene expression in which amplification of the target transcript relative to a reference gene transcript is used to compare relative expression. Great care needs to be taken in experimental design particularly in the selection of the house-keeping gene (Kozera \& Rapacz, 2013). This is particularly pertinent in the brain when analyzing mitochondrial gene expression, where the mtDNA is essentially an independently replicating genome, and suitable nuclear DNA-encoded reference genes are not well validated. Preliminary experiments to determine whether expression varied with SERT genotype showed that the routinely used house-keeping genes Hprt1 and Gapdh were adequate for analysis of gene expression in the FC but not in the $\mathrm{Cb}$ (Figures S4 and S5).

Next, we evaluated expression of the mtDNA gene for the respiratory complex subunits for Complex I (NADH dehydrogenase; $m t-N d 1, m t-N d 4)$, Complex IV (cytochrome oxidase; mt-Co1, mt-Co3), Complex V, (ATP synthase; mt-Atp8), and two nuclear encoded genes for Complex I (Ndufa9 and Ndufs2). Figure S2 shows that in female animals the SERT HETs showed reduced expression for $m t-N d 1$, $m t-$ Nd4, Ndufa9, mt-Co3, mt-Atp8 ( $p<0.05)$, and Ndufs2 $(p<0.01)$, but 
not for $m t-C o 1$ in the FC. In contrast, no significant differences were found for any of these transcripts for male HET animals (Figure S3). We interpret these findings with caution because of the inherent limitations for qRT-PCR when analyzing expression of mtDNA encoded genes.

Many of the limitations of qRT-PCR can be overcome by selfiedigital droplet PCR (ddPCR). Selfie-ddPCR is a powerful technique capable of measuring absolute gene expression without the use of reference genes as required by conventional qRT-PCR (Podlesniy \& Trullas, 2017). We used selfie-ddPCR to quantify mtDNA copy number and mRNA expression of $m t-N d 1$ and Ndufa9 in the $\mathrm{FC}$ and $\mathrm{Cb}$ of male and female SERT WT and HET rats. By normalizing transcript abundance to copies of the nuclear or mitochondrial genome, selfieddPCR measures absolute transcript abundance without the confounding effects of variations in expression of the reference genes.

Figure 1 shows unique genotype- and sex-dependent differences in mtDNA copy number in both the FC and $\mathrm{Cb}$. In the FC (Figure 1a), males and females appeared to show unique genotyperelated trends in mtDNA copy number, however, two-way ANOVA showed that the genotype*sex interaction did not reach statistical significance $(F(1,12)=3.64, p=0.081)$. As there was no significant interaction, the main effects were considered, showing mtDNA copy number was significantly greater for males $(F(1,12)=7.23$, $p=0.020)$, but there were no genotype-related differences $(F(1$, $12)=1.00, p=0.337)$. In the $\mathrm{Cb}$ (Figure $1 \mathrm{~b}$ ), two-way ANOVA also showed that there was no significant sex*genotype interaction $(F(1,15)=0.476, p=0.501)$, as both males and females show a similar trend of lower mtDNA copy number in HET animals. This is supported by the main effects which show that there were no sex-related differences $(F(1,15)=3.94, p=0.066)$, but that mtDNA copy number was significantly reduced in HET animals relative to WT $(F(1,15)=7.04, p=0.018)$.

Figure 2 shows expression of $\mathrm{mt}-\mathrm{Nd} 1$ in the $\mathrm{FC}$ and $\mathrm{Cb}$ of male and female rats relative to mtDNA copy number (panels $a$ and $b$ ) and also relative to the genome (panels $\mathrm{d}$ and e). In the FC, $m t-\mathrm{Nd} 1$ expression per mtDNA copy was reduced in HET females relative to WT but increased in HET males relative to WT (Figure 2a). As genotype-related differences were distinct in males and females, two-way ANOVA showed a significant genotype*sex interaction $(F(1,12)=12.5, p=0.004)$. The simple main effects were then considered, showing that expression was significantly reduced in female HETs relative to WT $(F(1,6)=18.8, p=0.005)$, but there were no genotype-related differences in males $(F(1,6)=1.14, p=0.327)$. Furthermore, expression was significantly greater in female WT animals relative to male $(F(1,6)=17.5, p=0.006)$, however, there were no sex-related differences for HET animals $(F(1,6)=0.98, p=0.361)$. In contrast, $m t-N d 1$ expression per mtDNA copy did not differ by genotype or sex in the $\mathrm{Cb}$. Two-way ANOVA showed that there was no genotype*sex interaction $(F(1,16)=0.50, p=0.491)$, with neither $\operatorname{sex}(F(1,16)=0.76, p=0.398)$ nor genotype $(F(1,16)=0.73$, $p=0.404$ ) significantly influencing expression.

As there were both genotype and sex differences in mtDNA copy number (Figure 1) and $m t-N d 1$ expression per mtDNA copy (Figure $2 \mathrm{a}, \mathrm{b}$ ) we examined $\mathrm{mt}-\mathrm{Nd} 1$ expression per cell by comparing expression relative to the nuclear genome. In the FC, $m t-N d 1$ expression per cell showed a very similar pattern to expression per mtDNA copy, and two-way ANOVA showed that there was a significant genotype*sex interaction $(F(1,12)=48.5, p<0.0001)$. Additionally, when comparing each sex and genotype, all of the simple main effects were also significant. Expression was significantly reduced in female $\operatorname{HETs}(F(1,6)=29.6, p=0.002)$ and significantly increased in male HETs relative to WT $(F(1,6)=21.7, p=0.003)$. Additionally, expression was significantly higher in female WT animals compared with male $(F(1,6)=26.7, p=0.002)$, whereas expression in male HETs was significantly greater than the female counterparts $(F(1,6)$ $=24.5, p=0.003$ ) animals. In the $\mathrm{Cb}$, there was no significant genotype*sex interaction for $m t-N d 1$ expression per cell $(F(1,16)=0.31$, $p=0.584)$. However, expression was significantly reduced in HET animals relative to $\mathrm{WT}(F(1,6)=10.5, p=0.005)$, and significantly increased in females compared to males $(F(1,6)=6.95, p=0.018)$.
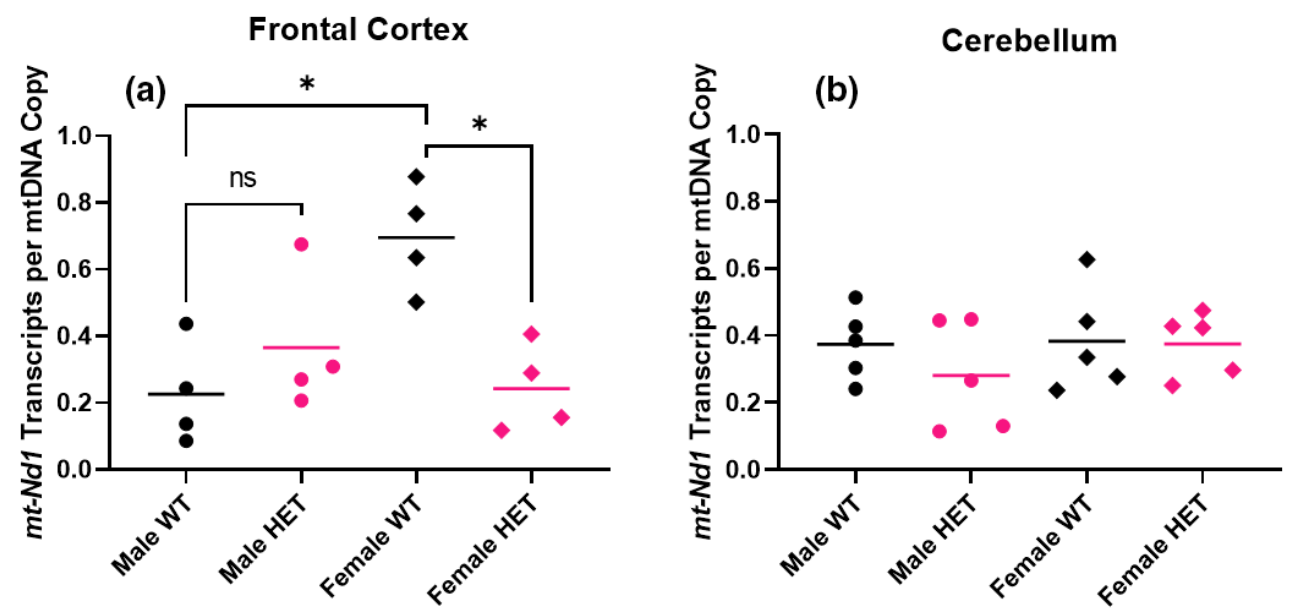

FIGURE 1 Sex- and genotype-dependent changes in mtDNA copy number in the FC and Cb. (a) mtDNA copy number is significantly reduced in female $(n=8)$ animals relative to male $(n=8)$ in the $F C(F(1,12)=7.23, p=0.020) .{ }^{*} p<0.05$. (b) mtDNA copy number is significantly reduced in HET $(n=10)$ animals relative to $\mathrm{WT}(n=10)$ in the $\mathrm{Cb}(F(1,15)=7.04, p=0.018) .{ }^{*} p<0.05$ 

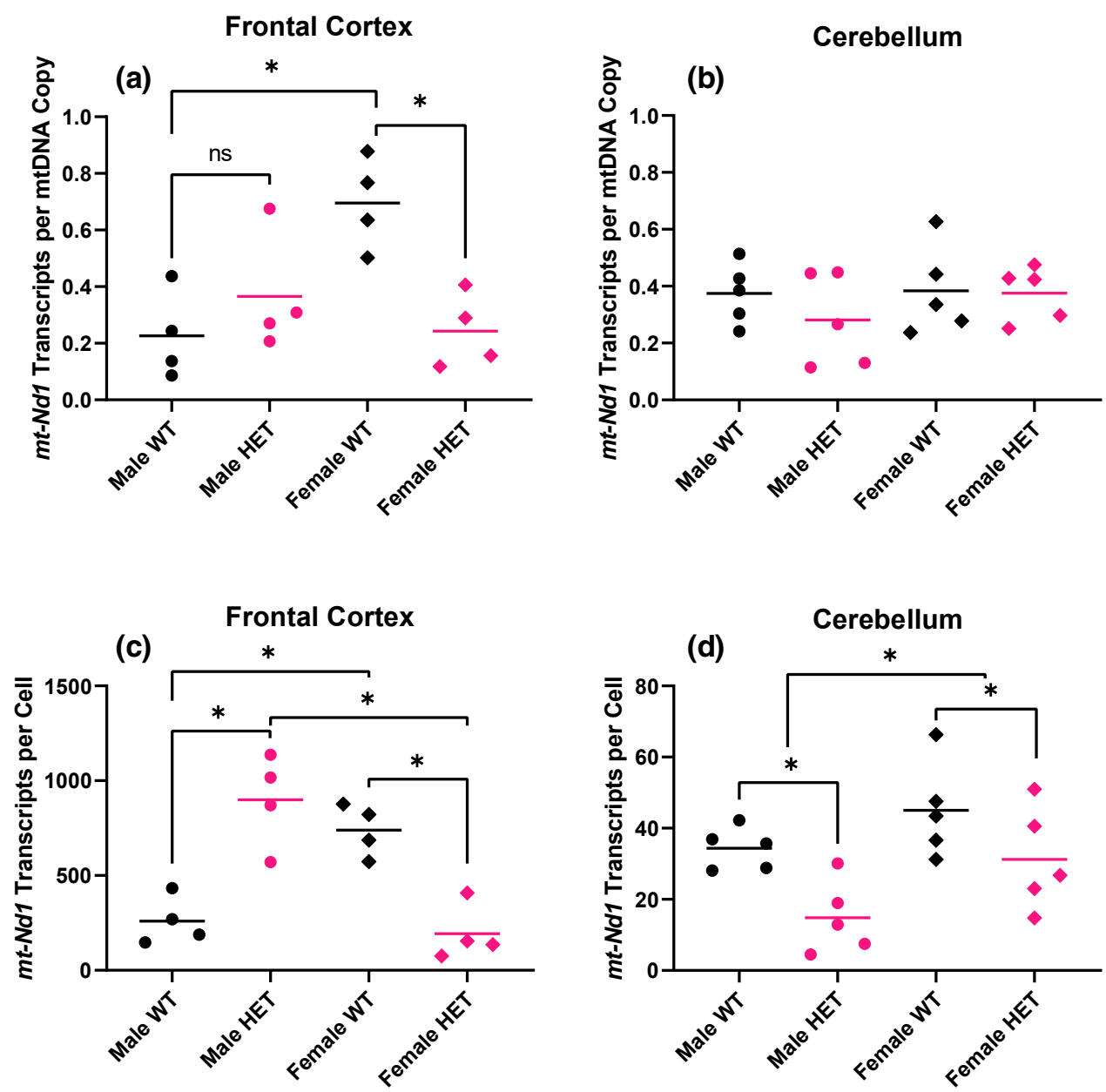

FIGURE 2 Sex- and genotype-dependent differences in mRNA expression of $m t-N d 1$. (a) mt-Nd1 expression per mtDNA copy in the FC ( $n=4$ per group). There was a significant genotype*sex interaction $(F(1,12)=12.5, p=0.004)$, expression was reduced in female HETs relative to WT $(n=4)(F(1,6)=18.8, p=0.005)$, and expression was significantly higher in female WT relative to male $(n=4)(F(1,6)=17.5$, $p=0.006$ ). ${ }^{*} p<0.0125$. (b) $m t-N d 1$ expression per mtDNA copy in the $\mathrm{Cb}$ ( $n=5$ per group). (c) $m t-N d 1$ expression per cell in the FC. There was a significant genotype* sex interaction $(F(1,12)=48.5, p<0.0001)$, expression was increased in male HET $(F(1,6)=21.7, p=0.003)$ and decreased in female HETs relative to $\mathrm{WT}(F(1,6)=29.6, p=0.002)$. Expression was significantly increased in female WT $(F(1,6)=26.7$, $p=0.002$ ) and decreased in female HETs relative to males $(F(1,6)=24.5, p=0.003) .{ }^{*} p<0.0125$. (d) $m t-N d 1$ expression per cell in the Cb. There was no significant genotype*sex interaction $(F(1,16)=0.312, p=0.584)$. Expression was increased in female animals relative to male $(F(1,16)=6.95, p=0.018)$ and decreased in HET relative to $\mathrm{WT}(F(1,16)=10.5, p=0.005) .{ }^{*} p<0.05$

While mtDNA copy number was significantly higher in the FC of all animals compared to the $\mathrm{Cb}$ (Figure 1), the number of $\mathrm{mt}-\mathrm{Nd} 1$ transcripts per mtDNA copy was similar between the $\mathrm{FC}$ and $\mathrm{Cb}$ (Figure 2a,b). Because of this, mt-Nd1 transcripts per cell was significantly greater in the $\mathrm{FC}$ and interestingly, the magnitude of this difference varied with genotype and sex. The greatest difference in $m t-N d 1$ expression between brain regions was in male HET animals, where expression per cell in the FC was over 60 times that of the $\mathrm{Cb}$. In contrast to this, expression in the FC of male WTs was around seven times that of the $\mathrm{Cb}$. For females, $\mathrm{mt}-\mathrm{Nd} 1$ expression per cell was 16 and six times higher in the FC than the $\mathrm{Cb}$ for WT and HET animals, respectively.

Next, we sought to determine whether the genotype and sex differences in expression observed for mitochondrial encoded respiratory complex genes were recapitulated for the genome encoded respiratory subunits.

In the FC of both male and female animals, Ndufa9 expression followed a similar genotype-related trend to $\mathrm{mt}-\mathrm{Nd} 1$, with a small increase in male HETs and a small decrease in female HETs relative to WT (Figure 3). There was a significant genotype*sex interaction $(F(1,12)=8.51, p=0.013)$, however, there were no genotyperelated differences in expression for males $(F(1,6)=5.26$, $p=0.062)$ or females $(F(1,6)=3.37, p=0.116)$. While Ndufa9 expression did not differ significantly between male and female WT animals $(F(1,6)=0.039, p=0.849)$, expression in male HETs was significantly greater than the female counterparts $(F(1,6)=16.87$, $p=0.006)$. While the trends in Ndufa9 expression reflected that of $m t-N d 1$, this was to a much lesser degree for Ndufa9. This is shown in the ratio of $m t-N d 1$ : Ndufa9 transcripts per cell. For female 

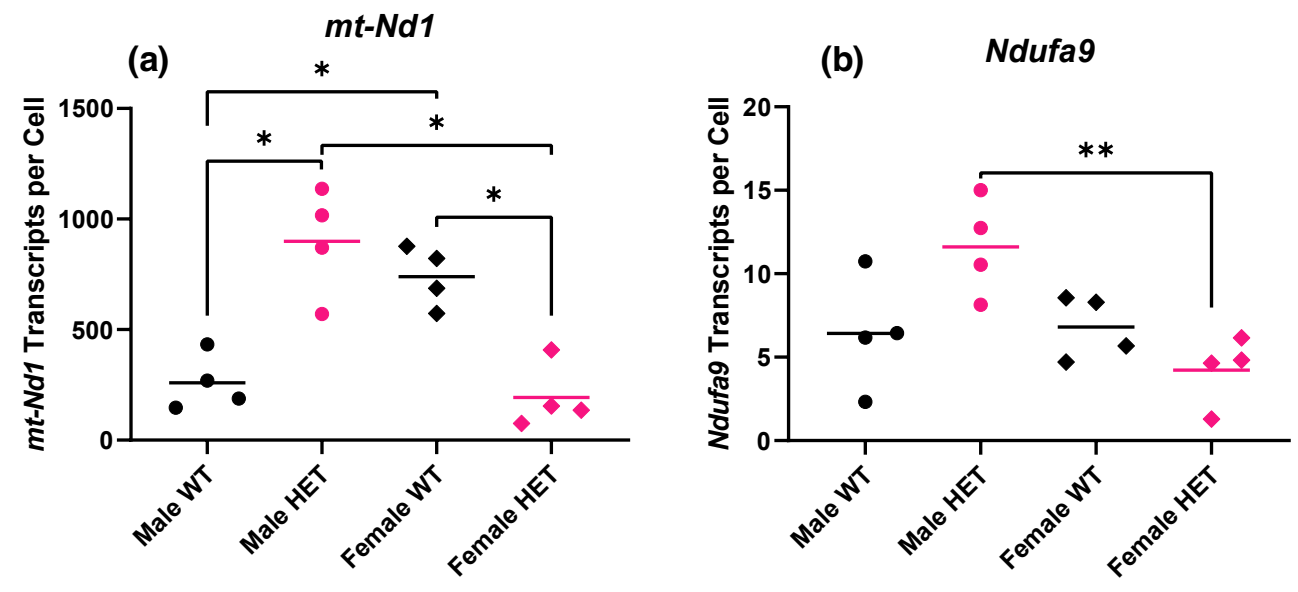

FIGURE 3 mRNA expression of $m t-N d 1$ and Ndufa9 in the FC of male and female SERT WT and HET animals. Genotype-related differences in expression of $\mathrm{mt}-\mathrm{Nd} 1$ and Ndufa9 follow similar trends, but to a lesser extent for Ndufa9

animals, this ratio was 109:1 in WTs and 46:1 in HETs whereas for males, the ratio was 40:1 in WT animals and 83:1 in HETs. This demonstrates that while reduced SERT expression influences the expression of both mitochondrial- and nuclear-encoded genes for respiratory chain subunits, the impact on mtDNA-encoded subunits is much more profound.

\section{3 | DISCUSSION}

The SERT HET and WT animals used in this study have been proposed to be a good model for human allelic variants found in the SERT promotor region (5-HTTLPR) which differ in the number of tandem repeats (Homberg, Olivier, et al., 2007; Olivier et al., 2008). The short (S) allele has been shown to have lower expression than the long (L) allele, with human S/S homozygotes being more sensitive to both positive and adverse life events than the L/L haplotype (van IJzendoorn et al., 2012). The rat SERT HET animals show reduced radioligand binding in the FC consistent with their haploinsufficiency (Homberg, Olivier, et al., 2007), yet behaviorally are very similar to WT animals (Homberg, Pattij, et al., 2007). Figure S1 shows that the cell bodies of the serotonergic raphe nuclei that project into the FC have approximate $70 \%$ less transcripts for SERT than the WT animals. The SERT haploinsufficient model we have used provides a good framework for investigating the effect of altered brain serotonin on mtDNA copy number and expression as serotonergic signaling through multiple 5 -HT receptors has been associated with mitochondrial biogenesis (Fanibunda et al., 2019; Scholpa et al., 2018; Simmons et al., 2019). The finding that SERT protein is associated with the outer membrane of the mitochondrion is intriguing as its potential function there is unclear (Haase et al., 2017; Mukherjee et al., 1998), as is the relative distribution between plasma membrane and mitochondria, or whether this differs by SERT genotype. The localization of monoamine oxidase $A$, which is an important enzyme for degrading intracellular serotonin on the mitochondrial outer membrane with SERT, is consistent with a potential role of mitochondria in serotonin homeostasis or mitochondrial activity being regulated by serotonin signaling. In this context we sought to determine whether the altered serotonergic signaling in SERT HET rats influenced mitochondrial abundance and mRNA expression in the brain. Here we report significant genotype related differences in mtDNA copy number and mRNA expression of $m t-N d 1$ and Complex IV and V mtDNA encoded subunits by qRT-PCR. These differences were pronounced in the FC and were highly sexually dimorphic.

Epidemiological studies have demonstrated significant sex differences in neuropsychiatric disorders. While women have a significantly greater lifetime risk for depression and anxiety, the suicide rate for depressed men is significantly higher than that for women (Freeman et al., 2017; Salk et al., 2017). Sex differences have also been associated with 5-HTTLPR genotype and likelihood of developing neuropsychiatric disorders. For women, research shows that the $\mathrm{S}$-allele confers a greater risk for developing depressive or anxiety disorders, especially in response to environmental stressors; however, this effect does not extend to men (reviewed in Gressier et al. (2016)). Additionally, sex-specific differences in serotonergic signaling and in the density of several 5 - $\mathrm{HT}$ receptors in the brain have been described (reviewed in Cosgrove et al. (2007)). The rate of 5-HT synthesis has also been shown to be $52 \%$ higher in the brain of males compared to females (Nishizawa et al., 1997), whereas women show higher levels of serotonin in the blood (Weiss et al., 2005). Mitochondrial function, particularly that of Complex I is also associated with neuropsychiatric disorders (Abramov \& Angelova, 2019), with respiratory chain function being enhanced in the brains of female rodents, in contrast to the mitochondria in male brains that produce significantly more reactive oxygen species (Borrás et al., 2003; Demarest \& McCarthy, 2015; Ruszkiewicz et al., 2019).

We speculate that the changes in copy number and gene expression seen in SERT HET animals are related to altered serotonergic signaling as well as SERT activity on the mitochondrial membrane, 
and that the reduction in expression of components of Complex I (NADH dehydrogenase) reduce mitochondrial electron transport and hence ATP synthesis. This hypothesis is consistent with the role of Complex I being the rate limiting enzyme for mitochondrial electron transport, and hence ATP synthesis (Sharma et al., 2009). While the functional significance of the changes in expression of $\mathrm{mt}-\mathrm{Nd} 1$ are unclear, it is reasonable to speculate that increased $\mathrm{mt}-\mathrm{Nd} 1$ expression leads to increased mitochondrial oxidative phosphorylation and higher ATP levels. This should be further investigated with experiments to assess functional changes in respiratory complex activity and oxygen consumption. A recent study by Horváth et al identified the purinergic ion channel $\mathrm{P} 2 \mathrm{rx} 7$, that is agonized by ATP, is a driver of autism-like behavior in a mouse model following poly I:C treatment (Horváth et al., 2019). Clearly the role of mitochondrial ATP production and activation of the purine receptor $\mathrm{P} 2 \mathrm{rx} 7$ in autism requires further investigation.

Studies in SERT knockout mice have shown that decreased SERT expression results in reduced expression of the 5-HT1A autoreceptor in the DRN, with a more extensive reduction in female animals ( $\mathrm{Li}$ et al., 2000). Altered expression of $5-\mathrm{HT} 2 \mathrm{~A}$ and $5-\mathrm{HT} 2 \mathrm{C}$ receptors has also been demonstrated in the brains of female SERT knockout mice, with changes being unique to the brain region (Li et al., 2003). While sexual dimorphism has been demonstrated in mitochondrial function, serotonergic signaling, and changes in 5-HT receptor expression in SERT knockout mice, the opposing trends in male and female HET animals are striking. Although it is unknown whether the changes in $\mathrm{mt}-\mathrm{Nd} 1$ expression are also associated with changes in mitochondrial function, it has been demonstrated that there is a strong correlation between mtDNA copy number, mitochondrial mRNA expression, and respiratory capacity (D'Erchia et al., 2015). Given the magnitude of the changes in expression, with $\mathrm{mt}-\mathrm{Nd} 1 \mathrm{ex}-$ pression in female HETs being approximately half that of WT, it is likely that these are of functional significance. The reduction in $\mathrm{mt}$ Nd1 expression in the FC of female HETs is therefore particularly significant given that MDD, which is more common in females, is also associated with reduced mitochondrial activity in the brain.

In the FC, mtDNA copy number and mRNA expression followed opposing genotype-related trends for males and females. Where mt$\mathrm{Nd} 1$ expression was significantly reduced in female HETs relative to WT, mtDNA copy number and $m t-N d 1$ expression were increased in male HETs. Interestingly, the expression of Ndufa9, a nuclear-encoded gene for complex I, followed the same trend. This suggests that the effect of SERT on mitochondrial gene expression also extend to nuclear encoded components, albeit to a lesser extent. The comparison in Figure 3 between transcript abundance of core components of Complex I that are either mtDNA encoded (mt-Nd1) or nuclear encoded (Ndufa9) is intriguing. While the expression of Ndufa9 follows the same trends as that for $m t-N d 1$ with respect to sex and genotype, the mRNA expressions levels are approximately 100 -fold less. It is not obvious how the appropriate stoichiometry of protein expression is achieved given the vast difference in transcript abundance and the required equimolar amounts of each protein in the functional respiratory complex. Reduced SERT expression has been shown to result in increased extracellular 5-HT, which likely influences signaling through pre- and post-synaptic 5-HT receptors (Mathews et al., 2004). Given that signaling through 5-HT2A, 5-HT1F, and 5-HT7 receptors has been shown to promote mitochondrial biogenesis, it is possible that the increased extracellular 5-HT in the HET animals would lead to increased signaling through these receptors, promoting mitochondrial biogenesis. While reductions in mtDNA copy number and mRNA expression were seen in the male FC, the opposite is observed in the $\mathrm{Cb}$ and the female FC. This highlights that there are likely additional compensatory mechanisms which are yet to be understood, potentially involving changes in expression of 5 - $\mathrm{HT}$ receptors.

In addition to genotype related differences, this study also demonstrated significant differences in mtDNA copy number and mRNA expression between brain regions. mtDNA copy number differed significantly between the $\mathrm{FC}$ and the $\mathrm{Cb}$, being around 10 times higher in the FC, supporting similar findings in the mouse brain (Fuke et al., 1807). However, expression of $\mathrm{mt}-\mathrm{Nd} 1$ per mitochondrial copy was comparatively consistent across brain regions. Interestingly, genotype-related changes in $\mathrm{mt}-\mathrm{Nd} 1$ expression per cell had different origins in the two brain regions. In the FC, differences in $\mathrm{mt}-\mathrm{Nd} 1$ expression per cell were driven by changes in both mtDNA copy number and the expression level of $m t-N d 1$ per mtDNA copy, whereas in the $\mathrm{Cb}$, changes in $\mathrm{mt}-\mathrm{Nd} 1$ expression were largely driven by reduced transcription of the mitochondrial genome.

Although the cerebellum largely functions to support motor control, it also has a lesser appreciated role in mood regulation (Depping et al., 2018). In particular, lobule VII exclusively communicates with areas involved with emotional processing and higher cognitive function (Buckner et al., 2011). Whole $\mathrm{Cb}$ homogenate was used in this study, so it is impossible to know whether changes in mtDNA abundance and expression are region specific within the $\mathrm{Cb}$. There is, however, significant serotonergic innervation throughout the $\mathrm{Cb}$, and it is evident that mitochondria in the cerebellum are influenced by reduced SERT expression. It is interesting that the strong sexual dimorphism seen in the FC did not extend to the $\mathrm{Cb}$; however, it is possible that investigation of specific regions within the $\mathrm{Cb}$ would identify further differences.

Both mitochondrial dysfunction and altered serotonergic signaling have been implicated in the pathophysiology of neurodevelopmental and neuropsychiatric disorders, and this study demonstrates a connection between these two aspects. Given that reduced brain mitochondrial function has been associated with MDD (Klinedinst \& Regenold, 2015; Streck et al., 2014; Videbech, 2000) and elevated mtDNA copy number with ASD (Chen et al., 2015; Yoo et al., 2017), and that both these disorders show a strong sex bias in prevalence, the impact of SERT genotype on mitochondrial gene expression we report in this study is significant. $\mathrm{mt}-\mathrm{Nd} 1$ expression was approximately halved in the FC of female HET rats and doubled in HET males relative to the WT, suggesting that mitochondrial activity, or at least maximal rate of ATP production may also be altered in this brain region. Without analysis of electron transfer and metabolic activity, it is unknown whether corresponding differences in mitochondrial oxidative phosphorylation are also present in the brains of 
these animals. However, changes in mtDNA copy number and mRNA expression are strongly associated with mitochondrial respiratory activity, suggesting that these changes are of biological relevance (Cieślik et al., 2021; D'Erchia et al., 2015; Malik \& Czajka, 2013). Whether these sex differences in expression lead to an alteration in mitochondrial-associated SERT requires further study and experimental validation. However, we speculate that altered serotonergic signaling as well as SERT associated with the mitochondrial membrane acts to regulate mitochondrial oxidative phosphorylation, thereby contributing to the sexual bias seen in the prevalence for some neuropsychiatric disorders (Figure 4) (Haase et al., 2017).

\section{4 | METHODS}

\section{1 | Animals}

The animals used in this study were male and female serotonin transporter knockout (SERT KO) rats (Slc6a41Hubr). SERT knockout rats were generated by ENU-mediated mutagenesis on a Wistar background and have been characterized extensively (Homberg, Olivier, et al., 2007; Olivier et al., 2008; Smits et al., 2006). SERT WT, HET, and homozygous knockout (HOM) male and female rats were bred from SERT HET $\times$ HET pairs. Five each of male and female WT and HET animals, as well as three female HOM animals were used in this study. Rats were housed in standard housing conditions in a temperature $\left(21^{\circ} \mathrm{C} \pm 2\right)$ and humidity $(55 \%-60 \%)$-controlled environment with 12-hr light-dark cycles and with access to chow and water ad libitum. Rats were sacrificed at postnatal day (PND) 60 by $\mathrm{CO}_{2}$ asphyxiation before rapid decapitation; dissections were completed using custom
3D printed brain blocks. Blades were placed at Bregma 3.2 and $1.2 \mathrm{~mm}$ to produce a $2 \mathrm{~mm}$ coronal section for the dissection of the frontal cortex (FC); an additional blade was placed at Bregma $-9.0 \mathrm{~mm}$ for the removal of the cerebellum. Final FC dissections included the primary motor cortex, primary and secondary somatosensory cortices. All experiments were approved by the Victoria University of Wellington Animal Ethics Committee (Approval Number 25766).

\section{2 | Lysis and reverse transcription}

Reverse transcription and absolute quantification of transcript abundance by selfie-ddPCR was performed as described in Podlesniy and Trullas (2017). Expression of complex I subunits encoded by the mitochondrial and nuclear genome was quantified using $\mathrm{mt}-\mathrm{Nd} 1$ and Ndufa9 as target genes. These genes were chosen as Complex I is an important regulator of mitochondrial redox activity and has been extensively used for determining mtDNA copy number. For this, total cellular DNA and RNA was isolated with the SingleShot Cell Lysis Kit (Bio-Rad, 172-8050; USA). Five milligram of tissue was homogenized before the addition of $72 \mu \mathrm{l}$ of cell lysis buffer and $1.5 \mu \mathrm{l}$ of proteinase $\mathrm{K}$. Samples were incubated for $10 \mathrm{~min}$ at room temperature followed by $5 \mathrm{~min}$ at $37^{\circ} \mathrm{C}$ and $5 \mathrm{~min}$ at $75^{\circ} \mathrm{C}$ for immediate use. Two microliter of the lysed sample was added to $9 \mu \mathrm{l}$ of $5 \mu \mathrm{M} \mathrm{mt-Nd1}$ and Ndufa9 reverse primer (Table 1), and samples were incubated in a thermocycler at $70^{\circ} \mathrm{C}$ for $1 \mathrm{~min}$ and brought to $4{ }^{\circ} \mathrm{C}$ at a slow ramp rate for primer annealing. For reverse transcription $5 \mu \mathrm{l}$ of the preannealed sample was then added to a mixture of $5.5 \mu \mathrm{l}$ nuclease-free water, $2 \mu \mathrm{l}$ RT buffer, $1 \mu \mathrm{l}$ dNTPs, $0.5 \mu \mathrm{l}$ RNaseOUT (ThermoFisher Scientific, 10777019; USA), $0.5 \mu \mathrm{l}$ DTT, $0.5 \mu \mathrm{l}$ Maxima H Minus
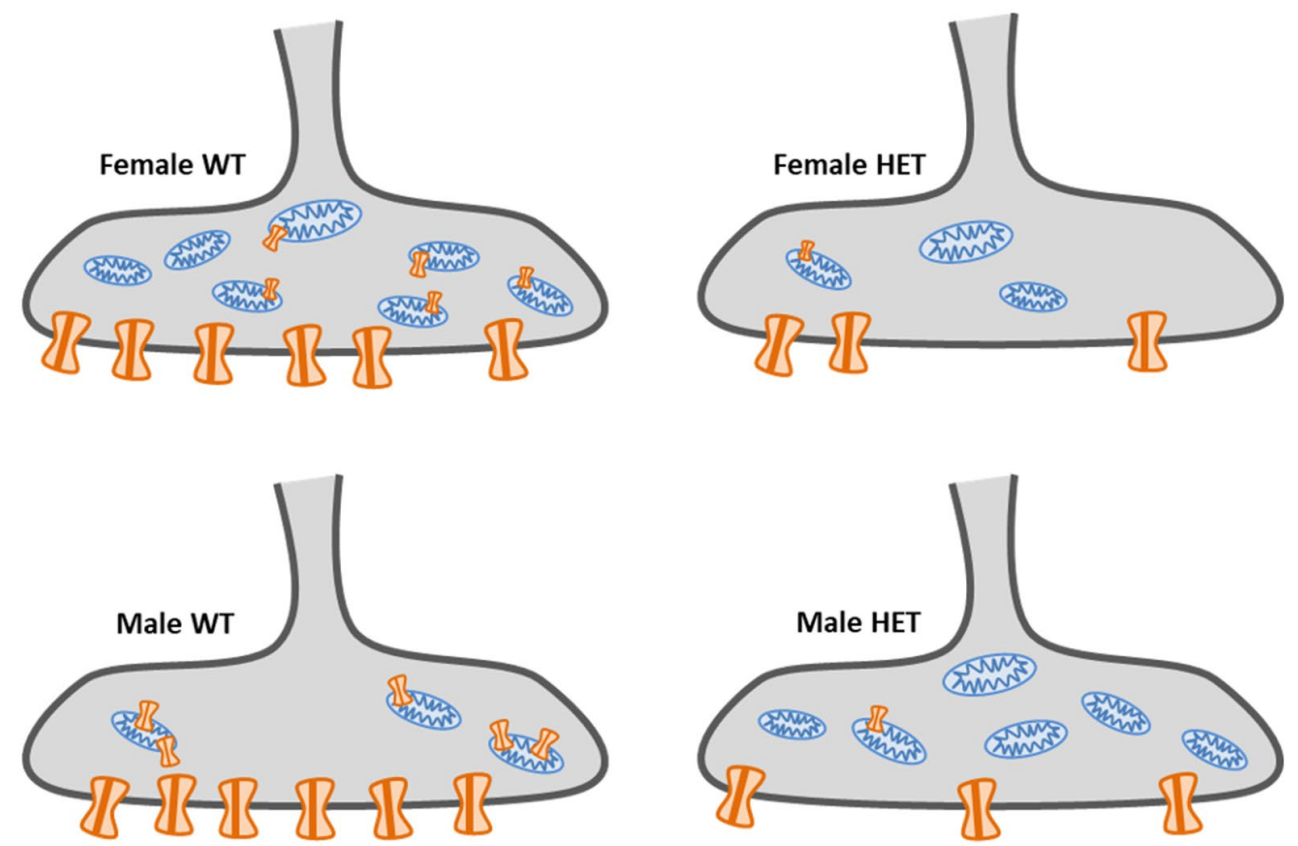

FIGURE 4 Depiction of unique sex- and genotype-dependent changes in mtDNA copy number and mitohcondrial mRNA expression in the FC of SERT WT and HET animals 
TABLE 1 Primer sequences used for selfie-ddPCR

\begin{tabular}{llll} 
& \multicolumn{2}{l}{ Primer sequence } & \\
\cline { 2 - 3 } Amplicon & Forward $\left(5^{\prime}-3^{\prime}\right)$ & Reverse $\left(5^{\prime}-3^{\prime}\right)$ & Product \\
mt-Nd1 & TTA ACG TCG AAT ACG CCG CA & AGC TGG TTG AGT ATA ATT CAG GGT & 144 bp \\
Ndufa9 & TGA GAT CGA GGA AAC CAA GCC & TAG AGC ACC CTC CTT CAG TAC A & 100 bp \\
\hline
\end{tabular}

reverse transcriptase (ThermoFisher Scientific, EP0751; USA) or RT buffer. Samples were incubated at $58^{\circ} \mathrm{C}$ for $30 \mathrm{~min}$, followed by $90^{\circ} \mathrm{C}$ for $3 \mathrm{~min}$ to terminate reverse transcription reactions. For optimal partitioning of DNA into droplets for ddPCR, a restriction digest with Hin6I (ThermoFisher Scientific, ER0481; USA) was undertaken prior to ddPCR. Two microliter of Hin6l was diluted in $8 \mu$ of the provided buffer, and $1 \mu \mathrm{l}$ of this dilution was added to the RT+/RT- reactions. Samples were incubated at $37^{\circ} \mathrm{C}$ for $1 \mathrm{hr}$ followed by $20 \mathrm{~min}$ at $65^{\circ} \mathrm{C}$. Samples were then diluted to a final volume of $100 \mu \mathrm{l}$ for ddPCR. A further 100-fold dilution was used for ddPCR amplification of mt-Nd1.

\section{3 | Selfie-ddPCR}

For ddPCR, $1 \mu$ l of the final RT sample was mixed thoroughly with 11 $\mu$ l of ddPCR EvaGreen Supermix (Bio-Rad, 186-4034; USA), $9 \mu$ of nuclease free water and $1.3 \mu \mathrm{l}$ of $2.5 \mu \mathrm{M}$ forward and reverse primers (Table 1) at room temperature. Twenty microliter of this mixture was then emulsified in $70 \mu$ l of Droplet Generation Oil for EvaGreen (Bio-Rad, 186-4005; USA). Forty microliter of the droplet emulsion was added to a 96-well plate for PCR amplification (C1000 Touch Thermal Cycler with 96-deep well reaction module, Bio-Rad; USA) using a $2^{\circ} \mathrm{C} / \mathrm{s}$ ramp rate with the following cycling conditions: $95^{\circ} \mathrm{C}$ for $5 \mathrm{~min}, 95^{\circ} \mathrm{C}$ for $30 \mathrm{~s}, 62^{\circ} \mathrm{C}$ for $1 \mathrm{~min}$ for 40 cycles, $4^{\circ} \mathrm{C}$ for $5 \mathrm{~min}$, and $90^{\circ} \mathrm{C}$ for $5 \mathrm{~min}$. No template controls containing all reagents minus template were included with each amplification to confirm the absence of contaminating DNA.

Droplet fluorescence analysis was undertaken using the QuantaSoft Analysis Pro software (Version 1.0.596, Bio-Rad; USA). Using a Poisson distribution, the number of copies per $\mu \mathrm{L}(\mathrm{N})$ is estimated by the software based on the number of positive and negative droplets. mtDNA copy number and mRNA expression were calculated:

$$
\begin{gathered}
m t-N d 1 \text { transcripts per mtDNA copy }=[\mathrm{NRT}+]-[\mathrm{NRT}-] /[\mathrm{NRT}-] \\
\begin{aligned}
m t-\mathrm{Nd} 1 \text { transcripts per cell } & =[(\mathrm{N} \text { mtDNART }+-\mathrm{NmtDNART}-) \times 100] \\
& -[\mathrm{NgDNART}-] /[\mathrm{NgDNART}-] \\
\text { Ndufa9 transcripts per cell }= & {[\mathrm{NRT}+]-[\mathrm{NRT}-] /[\mathrm{NRT}-] } \\
\mathrm{mtDNA} \text { copy number }= & {[\mathrm{N} \text { mtDNART }-\times 100]-[\mathrm{NgDNART}-] } \\
/ & {[\mathrm{NgDNART}-] }
\end{aligned}
\end{gathered}
$$

\section{4 | Statistical analysis}

All statistical analyses used in this research were conducted using IBM SPSS Statistics 25 (IBM; USA) and GraphPad Prism (version 8.4.1 for Windows, GraphPad Software; USA). Data were analyzed by two-way
ANOVA. If a statistically significant genotype*sex interaction $(p<0.05)$ was detected, the simple main effects were considered. For the simple main effects, the Bonferroni correction for multiple comparisons was applied and differences were considered statistically significant when $p<0.0125$. If there was not a significant genotype*sex interaction, the main effects were tested, and differences were considered statistically significant when $(p<0.05)$. To determine whether residuals were normally distributed, Q-Q plots were generated and assessed for each analysis. Levene's test was used to test the assumption of equal variance, with $p>0.05$ indicating equal variance; the assumption for equal variance was met for all analyses.

\section{DECLARATION OF TRANSPARENCY}

The authors, reviewers and editors affirm that in accordance to the policies set by the Journal of Neuroscience Research, this manuscript presents an accurate and transparent account of the study being reported and that all critical details describing the methods and results are present.

\section{CONFLICT OF INTEREST}

The authors declare no conflict of interest.

\section{AUTHOR CONTRIBUTIONS}

Conceptualization, D.J.D., B.N.T., and B.A.E.; Methodology, D.J.D. and B.N.T.; Investigation, D.J.D. and B.N.T.; Resources, D.J.D. and B.A.E.; Formal Analyses, B.N.T. and D.J.D.; Writing - Review \& Editing, B.N.T., D.J.D., and B.A.E.

\section{PEER REVIEW}

The peer review history for this article is available at https://publo ns.com/publon/10.1002/jnr.25010.

\section{DATA AVAILABILITY STATEMENT}

Data are available upon request to the corresponding author.

\section{REFERENCES}

Abramov, A. Y., \& Angelova, P. R. (2019). Cellular mechanisms of complex I-associated pathology. Biochemical Society Transactions, 47, 19631969. https://doi.org/10.1042/BST20191042

Allen, J., Romay-Tallon, R., Brymer, K. J., Caruncho, H. J., \& Kalynchuk, L. E. (2018). Mitochondria and mood: Mitochondrial dysfunction as a key player in the manifestation of depression. Frontiers in Neuroscience, 12, 386. https://doi.org/10.3389/ fnins.2018.00386

Bansal, Y., \& Kuhad, A. (2016). Mitochondrial dysfunction in depression. Current Neuropharmacology, 14, 610-618. https://doi. org/10.2174/1570159X14666160229114755 
Border, R., Johnson, E. C., Evans, L. M., Smolen, A., Berley, N., Sullivan, P. F., \& Keller, M. C. (2019). No support for historical candidate gene or candidate gene-by-interaction hypotheses for major depression across multiple large samples. American Journal of Psychiatry, 176, 376-387. https://doi.org/10.1176/appi. ajp.2018.18070881

Borrás, C., Sastre, J., García-Sala, D., Lloret, A., Pallardó, F. V., \& Viña, J. (2003). Mitochondria from females exhibit higher antioxidant gene expression and lower oxidative damage than males. Free Radical Biology and Medicine, 34, 546-552. https://doi.org/10.1016/s0891 $-5849(02) 01356-4$

Brown, G. W., \& Harris, T. O. (2008). Depression and the serotonin transporter 5-HTTLPR polymorphism: A review and a hypothesis concerning gene-environment interaction. Journal of Affective Disorders, 111, 1-12. https://doi.org/10.1016/j.jad.2008.04.009

Buckner, R. L., Krienen, F. M., Castellanos, A., Diaz, J. C., \& Yeo, B. T. T. (2011). The organization of the human cerebellum estimated by intrinsic functional connectivity. Journal of Neurophysiology, 106, 2322-2345. https://doi.org/10.1152/jn.00339.2011

Campbell, S., Marriott, M., Nahmias, C., \& MacQueen, G. M. (2004). Lower hippocampal volume in patients suffering from depression: A meta-analysis. American Journal of Psychiatry, 161, 598-607. https://doi.org/10.1176/appi.ajp.161.4.598

Chaji, D., Venkatesh, V. S., Shirao, T., Day, D. J., \& Ellenbroek, B. A. (2021). Genetic knockout of the serotonin reuptake transporter results in the reduction of dendritic spines in in vitro rat cortical neuronal culture. Journal of Molecular Neuroscience, 71(11), 2210-2218. https:// doi.org/10.1007/s12031-020-01764-9

Chen, S., Li, Z., He, Y., Zhang, F., Li, H., Liao, Y., Wei, Z., Wan, G., Xiang, X., Hu, M., Xia, K., Chen, X., \& Tang, J. (2015). Elevated mitochondrial DNA copy number in peripheral blood cells is associated with childhood autism. BMC Psychiatry, 15, 50. https://doi.org/10.1186/ s12888-015-0432-y

Cieślik, M., Gassowska-Dobrowolska, M.,Zawadzka,A., Frontczak-Baniewicz, M., Gewartowska, M., Dominiak, A., Czapski, G. A., \& Adamczyk, A. (2021). The synaptic dysregulation in adolescent rats exposed to maternal immune activation. Frontiers in Molecular Neuroscience, 13, 555290. https://doi.org/10.3389/fnmol.2020.555290

Cosgrove, K. P., Mazure, C. M., \& Staley, J. K. (2007). Evolving knowledge of sex differences in brain structure, function and chemistry. Biological Psychiatry, 62, 847-855. https://doi.org/10.1016/j.biops ych.2007.03.001

D'Erchia, A. M., Atlante, A., Gadaleta, G., Pavesi, G., Chiara, M., De Virgilio, C., Manzari, C., Mastropasqua, F., Prazzoli, G. M., Picardi, E., Gissi, C., Horner, D., Reyes, A., Sbisà, E., Tullo, A., \& Pesole, G. (2015). Tissue-specific mtDNA abundance from exome data and its correlation with mitochondrial transcription, mass and respiratory activity. Mitochondrion, 20, 13-21. https://doi.org/10.1016/j.mito.2014.10.005

Demarest, T. G., \& McCarthy, M. M. (2015). Sex differences in mitochondrial (dys)function: Implications for neuroprotection. Journal of Bioenergetics and Biomembranes, 47, 173-188. https://doi. org/10.1007/s10863-014-9583-7

Depping, M. S., Schmitgen, M. M., Kubera, K. M., \& Wolf, R. C. (2018). Cerebellar contributions to major depression. Frontiers in Psychiatry, 9, 634. https://doi.org/10.3389/fpsyt.2018.00634

Fanibunda, S. E., Deb, S., Maniyadath, B., Tiwari, P., Ghai, U., Gupta, S., Figueiredo, D., Weisstaub, N., Gingrich, J. A., Vaidya, A. D. B., Kolthur-Seetharam, U., \& Vaidya, V. A. (2019). Serotonin regulates mitochondrial biogenesis and function in rodent cortical neurons via the 5-HT2A receptor and SIRT1-PGC-1 alpha axis. Proceedings of the National Academy of Sciences of the United States of America, 116, 11028-11037. https://doi.org/10.1073/pnas.1821332116

Fattal, O., Budur, K., Vaughan, A. J., \& Franco, K. (2006). Review of the literature on major mental disorders in adult patients with mitochondrial diseases. Psychosomatics, 47, 1-7. https://doi.org/10.1176/ appi.psy.47.1.1
Ferrari, F., \& Villa, R. F. (2017). The neurobiology of depression: An integrated overview from biological theories to clinical evidence. Molecular Neurobiology, 54, 4847-4865. https://doi.org/10.1007/ s12035-016-0032-y

Freeman, A., Mergl, R., Kohls, E., Székely, A., Gusmao, R., Arensman, E., Koburger, N., Hegerl, U., \& Rummel-Kluge, C. (2017). A crossnational study on gender differences in suicide intent. BMC Psychiatry, 17, 234. https://doi.org/10.1186/s12888-017-1398-8

Fuke, S., Kubota-Sakashita, M., Kasahara, T., Shigeyoshi, Y., \& Kato, T. (1807). Regional variation in mitochondrial DNA copy number in mouse brain. Biochimica et Biophysica Acta, 2011, 270-274. https:// doi.org/10.1016/j.bbabio.2010.11.016

Gibbs, W. S., Collier, J. B., Morris, M., Beeson, C. C., Megyesi, J., \& Schnellmann, R. G. (2018). 5-HT1F receptor regulates mitochondrial homeostasis and its loss potentiates acute kidney injury and impairs renal recovery. American Journal of Physiology-Renal Physiology, 315, F1119-F1128. https://doi.org/10.1152/ajprenal.00077.2018

Gressier, F., Calati, R., \& Serretti, A. (2016). 5-HTTLPR and gender differences in affective disorders: A systematic review. Journal of Affective Disorders, 190, 193-207. https://doi.org/10.1016/j. jad.2015.09.027

Haase, J., Grudzinska-Goebel, J., Muller, H. K., Muenster-Wandowski, A., Chow, E., Wynne, K., Farsi, Z., Zander, J.-F., \& Ahnert-Hilger, G. (2017). Serotonin transporter associated protein complexes are enriched in synaptic vesicle proteins and proteins involved in energy metabolism and ion homeostasis. ACS Chemical Neuroscience, 8, 1101-1116. https://doi.org/10.1021/acsch emneuro.6b00437

Hollis, F., Kanellopoulos, A. K., \& Bagni, C. (2017). Mitochondrial dysfunction in autism spectrum disorder: Clinical features and perspectives. Current Opinion in Neurobiology, 45, 178-187. https://doi. org/10.1016/j.conb.2017.05.018

Homberg, J. R., De Boer, S. F., Raasø, H. S., Olivier, J. D. A., Verheul, M., Ronken, E., Cools, A. R., Ellenbroek, B. A., Schoffelmeer, A. N. M., Vanderschuren, L. J. M. J., De Vries, T. J., \& Cuppen, E. (2008). Adaptations in pre- and postsynaptic 5-HT1A receptor function and cocaine supersensitivity in serotonin transporter knockout rats. Psychopharmacology (Berl), 200, 367-380. https://doi.org/10.1007/ s00213-008-1212-x

Homberg, J. R., Olivier, J. D. A., Smits, B. M. G., Mul, J. D., Mudde, J., Verheul, M., Nieuwenhuizen, O. F. M., Cools, A. R., Ronken, E., Cremers, T., Schoffelmeer, A. N. M., Ellenbroek, B. A., \& Cuppen, E. (2007). Characterization of the serotonin transporter knockout rat: A selective change in the functioning of the serotonergic system. Neuroscience, 146, 1662-1676. https://doi.org/10.1016/j.neuro science.2007.03.030

Homberg, J. R., Pattij, T., Janssen, M. C. W., Ronken, E., De Boer, S. F., Schoffelmeer, A. N. M., \& Cuppen, E. (2007). Serotonin transporter deficiency in rats improves inhibitory control but not behavioural flexibility. European Journal of Neuroscience, 26, 2066-2073. https:// doi.org/10.1111/j.1460-9568.2007.05839.x

Horváth, G., Otrokocsi, L., Beko, K., Baranyi, M., Kittel, Á., Fritz-Ruenes, P. A., \& Sperlágh, B. (2019). P2X7 receptors drive poly(l:C) induced autism-like behavior in mice. Journal of Neuroscience, 39, 25422561. https://doi.org/10.1523/JNEUROSCI.1895-18.2019

Klinedinst, N. J., \& Regenold, W. T. (2015). A mitochondrial bioenergetic basis of depression. Journal of Bioenergetics and Biomembranes, 47, 155-171. https://doi.org/10.1007/s10863-014-9584-6

Kozera, B., \& Rapacz, M. (2013). Reference genes in real-time PCR. Journal of Applied Genetics, 54, 391-406. https://doi.org/10.1007/ s13353-013-0173-x

Kraus, C., Castren, E., Kasper, S., \& Lanzenberger, R. (2017). Serotonin and neuroplasticity-Links between molecular, functional and structural pathophysiology in depression. Neuroscience and Biobehavioral Reviews, 77, 317-326. https://doi.org/10.1016/j. neubiorev.2017.03.007 
Lesch, K.-P., Bengel, D., Heils, A., Sabol, S. Z., Greenberg, B. D., Petri, S., Benjamin, J., Müller, C. R., Hamer, D. H., \& Murphy, D. L. (1996). Association of anxiety-related traits with a polymorphism in the serotonin transporter gene regulatory region. Science, 274, 15271531. https://doi.org/10.1126/science.274.5292.1527

Levinstein, M. R., \& Samuels, B. A. (2014). Mechanisms underlying the antidepressant response and treatment resistance. Frontiers in Behavioural Neurosciences, 8, 208. https://doi.org/10.3389/ fnbeh.2014.00208

Li, Q., Wichems, C., Heils, A., Lesch, K. P., \& Murphy, D. L. (2000). Reduction in the density and expression, but not G-protein coupling, of serotonin receptors (5-HT1A) in 5-HT transporter knock-out mice: Gender and brain region differences. Journal of Neuroscience, 20, 7888-7895.

Li, Q., Wichems, C. H., Ma, L., Van de Kar, L. D., Garcia, F., \& Murphy, D. L. (2003). Brain region-specific alterations of 5-HT2A and 5-HT2C receptors in serotonin transporter knockout mice. Journal of Neurochemistry, 84, 1256-1265. https://doi.org/10.1046/j.1471-4159.2003.01607.x

MacQueen, G. M., Campbell, S., McEwen, B., Macdonald, K., \& Young, T. (2003). Course of illness, hippocampal function and volume in major depression. Biological Psychiatry, 53, 31S.

Malik, A. N., \& Czajka, A. (2013). Is mitochondrial DNA content a potential biomarker of mitochondrial dysfunction? Mitochondrion, 13 481-492. https://doi.org/10.1016/j.mito.2012.10.011

Mathews, T. A., Fedele, D. E., Coppelli, F. M., Avila, A. M., Murphy, D. L., \& Andrews, A. M. (2004). Gene dose-dependent alterations in extraneuronal serotonin but not dopamine in mice with reduced serotonin transporter expression. Journal of Neuroscience Methods, 140, 169-181. https://doi.org/10.1016/j.jneumeth.2004.05.017

Mukherjee, J., Das, M. K., Yang, Z. Y., \& Lew, R. (1998). Evaluation of the binding of the radiolabeled antidepressant drug, F-18fluoxetine in the rodent brain: An in vitro and in vivo study. Nuclear Medicine and Biology, 25, 605-610. https://doi.org/10.1016/S0969 -8051(98)00043-2

Muller, C. L., Anacker, J., \& Veenstra-Vanderweele, J. (2016). The serotonin system in autism spectrum disorder: From biomarker to animal models. Neuroscience, 321, 24-41. https://doi.org/10.1016/j. neuroscience.2015.11.010

Nishizawa, S., Benkelfat, C., Young, S. N., Leyton, M., Mzengeza, S., de Montigny, C., Blier, P., \& Diksic, M. (1997). Differences between males and females in rates of serotonin synthesis in human brain. Proceedings of the National Academy of Sciences of the United States of America, 94, 5308-5313.

Olivier, J. D. A., Van Der Hart, M. G. C., Van Swelm, R. P. L., Dederen, P. J., Homberg, J. R., Cremers, T., Deen, P. M. T., Cuppen, E., Cools, A. R., \& Ellenbroek, B. A. (2008). A study in male and female 5-HT transporter knockout rats: An animal model for anxiety and depression disorders. Neuroscience, 152, 573-584. https://doi.org/10.1016/j. neuroscience.2007.12.032

Podlesniy, P., \& Trullas, R. (2017). Absolute measurement of gene transcripts with selfie-digital PCR. Scientific Reports, 7, 8328. https:// doi.org/10.1038/s41598-017-08270-w

Rossi, M.J., \& Pekkurnaz, G. (2019). Powerhouse of the mind: Mitochondrial plasticity at the synapse. Current Opinion in Neurobiology, 57, 149155. https://doi.org/10.1016/j.conb.2019.02.001

Ruszkiewicz, J. A., Miranda-Vizuete, A., Tinkov, A. A., Skalnaya, M. G., Skalny, A. V., Tsatsakis, A., \& Aschner, M. (2019). Sex-Specific differences in redox homeostasis in brain norm and disease. Journal of Molecular Neuroscience, 67, 312-342. https://doi.org/10.1007/ s12031-018-1241-9

Salk, R. H., Hyde, J. S., \& Abramson, L. Y. (2017). Gender differences in depression in representative national samples: Meta-analyses of diagnoses and symptoms. Psychological Bulletin, 143, 783-822. https://doi.org/10.1037/bul0000102

Scholpa, N. E., Lynn, M. K., Corum, D., Boger, H. A., \& Schnellmann, R. G. (2018). 5-HT1F receptor-mediated mitochondrial biogenesis for the treatment of Parkinson's disease. British Journal of Pharmacology, 175, 348-358. https://doi.org/10.1111/bph.14076

Sharma, L. K., Lu, J., \& Bai, Y. (2009). Mitochondrial respiratory complex I: Structure, function and implication in human diseases. Current Medicinal Chemistry, 16, 1266-1277.

Siddiqui, M. F., Elwell, C., \& Johnson, M. H. (2016). Mitochondrial dysfunction in autism spectrum disorders. Autism-Open Access, 6, 1000190. https://doi.org/10.4172/2165-7890.1000190

Simmons, E. C., Scholpa, N. E., Cleveland, K. H., \& Schnellmann, R. G. (2019). 5-HT1F receptor agonist induces mitochondrial biogenesis and promotes recovery from spinal cord injury. Journal of Pharmacology and Experimental Therapeutics, 372(2), 216-223. https://doi.org/10.1124/jpet.119.262410

Smits, B. M. G., Mudde, J. B., van de Belt, J., Verheul, M., Olivier, J., Homberg, J., Guryev, V., Cools, A. R., Ellenbroek, B. A., Plasterk, R. H. A., \& Cuppen, E. (2006). Generation of gene knockouts and mutant models in the laboratory rat by ENU-driven target-selected mutagenesis. Pharmacogenetics and Genomics, 16, 159-169. https:// doi.org/10.1097/01.fpc.0000184960.82903.8f

Streck, E. L., Goncalves, C. L., Furlanetto, C. B., Scaini, G., Dal-Pizzol, F., \& Quevedo, J. (2014). Mitochondria and the central nervous system: Searching for a pathophysiological basis of psychiatric disorders. Revista Brasileira de Psiquiatria, 36, 156-167. https://doi. org/10.1590/1516-4446-2013-1224

Valenti, D., de Bari, L., Vigli, D., Lacivita, E., Leopoldo, M., Laviola, G., Vacca, R. A., \& De Filippis, B. (2017). Stimulation of the brain serotonin receptor 7 rescues mitochondrial dysfunction in female mice from two models of Rett syndrome. Neuropharmacology, 121, 7988. https://doi.org/10.1016/j.neuropharm.2017.04.024

van IJzendoorn, M. H., Belsky, J., \& Bakermans-Kranenburg, M. J. (2012). Serotonin transporter genotype 5HTTLPR as a marker of differential susceptibility? A meta-analysis of child and adolescent gene-byenvironment studies. Translational Psychiatry, 2, e147. https://doi. org/10.1038/tp.2012.73

Videbech, P. (2000). PET measurements of brain glucose metabolism and blood flow in major depressive disorder: A critical review. Acta Psychiatrica Scandinavica, 101, 11-20. https://doi.org/10.1034/ j.1600-0447.2000.101001011.x

Weiss, L. A., Abney, M., Cook, E. H., \& Ober, C. (2005). Sexspecific genetic architecture of whole blood serotonin levels. American Journal of Human Genetics, 76, 33-41. https://doi. org/10.1086/426697

Yohn, C. N., Gergues, M. M., \& Samuels, B. A. (2017). The role of 5-HT receptors in depression. Molecular Brain, 10, 28. https://doi. org/10.1186/s13041-017-0306-y

Yoo, H. J., Park, M., \& Kim, S. A. (2017). Difference in mitochondrial DNA copy number in peripheral blood cells between probands with autism spectrum disorders and their unaffected siblings. World Journal of Biological Psychiatry, 18, 151-156. https://doi. org/10.1080/15622975.2016.1234069

\section{SUPPORTING INFORMATION}

Additional supporting information may be found in the online version of the article at the publisher's website.

FIGURE S1 (A) Relative mRNA expression of SERT in the dorsal raphe of SERT WT, HET, and HOM animals. Relative expression is shown

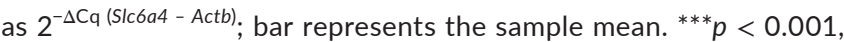
${ }^{* *} p<0.01,{ }^{*} p<0.05$. (b-c) Normalised melt curves and difference curves for SERT WT, HET, and HOM DNA and cDNA

FIGURE S2 Relative mRNA expression of mtDNA- and gDNAencoded genes for complex I, complex IV, and ATP synthase in the FC of female SERT WT and HET rats. Relative expression is shown as 
$2^{-\Delta \mathrm{Cq} \text { (Target Gene-Reference) }}$; bar represents the sample mean. ${ }^{*} p<0.05$, ${ }^{* *} p<0.01$

FIGURE S3 Relative mRNA expression of mtDNA- and gDNAencoded genes for complex I, complex IV, and ATP synthase in the FC of male SERT WT and HET rats. Relative expression is shown as $2^{-\Delta \mathrm{Cq} \text { (Target Gene - Reference) }}$

FIGURE S4 Relative mRNA expression of mtDNA- and gDNAencoded genes for complex $\mathrm{I}$ in the $\mathrm{Cb}$ of male (O) and female SERT WT, HET, and HOM rats. Relative expression is shown as

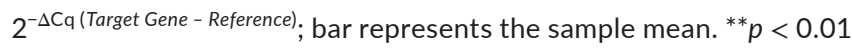
FIGURE S5 Relative mRNA expression of mtDNA- and gDNAencoded genes for complex I in the FC of male (O) and female $(>)$ SERT WT, HET, and HOM rats. Relative expression is shown as

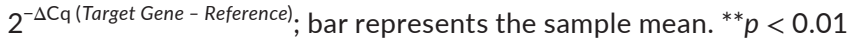

TABLE S1 Primer sequences used for RT-qPCR and HRMA

TABLE S2 Results from student's t-test analyses of mRNA expression in the FC of male and female SERT WT and HET animals

Transparent Science Questionnaire for Authors

How to cite this article: Thorne, B. N., Ellenbroek, B. A., \& Day, D. J. (2022). The serotonin reuptake transporter modulates mitochondrial copy number and mitochondrial respiratory complex gene expression in the frontal cortex and cerebellum in a sexually dimorphic manner. Journal of Neuroscience Research, 00, 1-11. https://doi.org/10.1002/ $\underline{\text { jnr.25010 }}$ 